\title{
Considerações sobre pulgas (Siphonaptera) da raposa Cerdocyon thous (Canidae) da área endêmica de leishmaniose visceral de Jacobina, Bahia, Brasil
}

\author{
Notes on fleas (Siphonaptera) of the fox Cerdocyon thous (Canidae) from \\ the visceral leishmaniasis endemic area of Jacobina, Bahia, Brazil
}

Elúzio J.L. Cerqueira, Evandro M. Silva, Adriano F. Monte-Alegre e Ítalo A. Sherlock ${ }^{1}$

Resumo No período de julho a setembro de 1998 foram coletadas 152 pulgas em 18 exemplares da raposa Cerdocyon thous capturados na área endêmica de leishmaniose visceral de Jacobina, Estado da Bahia. As pulgas foram identificadas como: 136 Rhopalopsyllus lutzi lutzi, 11 Pulex irritans, 2 Ctenocephalides canis, 1 Ctenocephalides felis felis e 2 Xenopsylla cheopis.

Palavras-chaves: Cerdocyon thous. Siphonaptera. Pulgas. Raposa. Canidae.

Abstract From July to September of 1998, 152 fleas were collected from 18 specimens of the fox Cerdocyon thous captured in the visceral leishmaniasis endemic area of Jacobina, State of Bahia, Brazil. The fleas were identified as: 136 Rhopalopsyllus lutzi lutzi, 11 Pulex irritans, 2 Ctenocephalides canis, 1 Ctenocephalides felis felis and 2 Xenopsylla cheopis.

Key-words: Cerdocyon thous. Siphonaptera. Fleas. Rox. Canidae.

Cerdocyon thous (Linnaeus, 1789) é a única espécie de raposa encontrada naturalmente infectada pela Leishmania chagasi Cunha \& Chagas, 1936, na região neotropical ${ }^{4}$. $\mathrm{Na}$ nomenclatura de Canideos Lycalopex vetulus (Lund,1842) é considerado sinônimo de Dusicyon vetulus (Lund,1842) ${ }^{4}$. Após minucioso estudo comparativo dos crânios e da morfologia dental de exemplares de raposas coletadas no Nordeste do Brasil e de conformidade com a faixa de distribuição geográfica das espécies foi concluido que a raposa existente no Nordeste é a Cerdocyon thous idêntica a encontrada na ilha de Marajót. A espécie Dusicyon vetulus só existe no Brasil Central.

Exemplares de Cerdocyon thous foram encontrados naturalmente infectados por Leishmania chagasi, em áreas endêmicas do Nordeste do Brasil, onde o flebótomo Lutzomyia longipalpis (Lutz \& Neiva, 1912) é o vetor comprovado da protozoose ${ }^{16}$.

$\mathrm{Na}$ llha de Marajó, Estado do Pará, onde vários exemplares desse canídeo também foram encontrados naturalmente infectados com a mesma leishmânia, uma associação foi observada entre o referido flebótomo e o canídeo ${ }^{9}$, tudo indicando que o flebótomo seja o principal transmissor da doença. Ainda que Lima ${ }^{10}$ tivesse admitido que as pulgas pudessem transmitir leishmanioses (Calazar e botão do Oriente) e que Alencar ${ }^{1}$ tivesse mencionado a infecção da pulga do cão pela Leishmania infantum Nicolle,1908, bem como a transmissão da leishmaniose canina por meio desse inseto, até o momento, não há qualquer dado confirmado na literatura pertinente (Bibikova $^{3}$, Daoust ${ }^{5}$ ). Assim é que Sherlock ${ }^{12}$ ao examinar exemplares de pulgas retiradas de cães com leishmaniose de zona endêmica, também

Laboratórios de Parasitologia e Entomologia do Centro de Pesquisas Gonçalo Moniz da Fundação Oswaldo Cruz e Faculdade de Farmácia e Bioquímica da Universidade Federal da Bahia, Salvador, BA.

Endereço para correspondência: Prof. Eluzio J.L. Cerqueira. CPqGM/FIOCRUZ. Rua Valdemar Falcão 121, Brotas, Salvador, BA. Tel: 5571 356-8785, Fax: 5571 356-4292.

Recebido para publicação em 1/12/98. 
não encontrou exemplares infectados. Infecções de pulgas por flagelados Herpetomonas ctenocephali foram noticiadas por Alencar ${ }^{1}$.

Vale ressaltar que Pacheco et al ${ }^{11}$ publicaram recentemente um interessante caso de um paciente com síndrome da imunodeficiência adquirida que apresentava sintomatologia de leishmaniose visceral mas que, no entanto, era provocada pela infecção por um tripanosomatideo monoxeno, a Leptomona pulexsimulantis Beard, Butler \& Orshar, $1989^{2}$, flagelado este que vive normalmente no intestino da pulga Pulex simulans. Os referidos autores ainda apresentaram referências bibliográficas sobre outros casos semelhantes.

Aproveitando a oportunidade em que estão sendo feito exames para o encontro de reservatórios silvestres de leishmaniose visceral, algumas pulgas retiradas de exemplares da raposa Cerdocyon thous capturadas na área endêmica do Município de Jacobina, Estado da Bahia, durante o período de julho a setembro de 1998, foram estudadas e os resultados da identificação especifica, são aqui apresentados.

Após anestesia geral dos canídeos para procedimento de exames parasitológicos, aquelas pulgas que eram visualizadas, eram retiradas com pinças e preservadas em álcool a $70^{\circ}$. Considerando que a coleta dos insetos era feita instantaneamente apenas enquanto durava o efeito anestésico nos mamíferos, um expressivo número de pulgas permanecia nos animais. Após tratamento em hidróxido de potássio a 10\% e procedimento convencional, as pulgas eram montados em bálsamo do Canadá e identificadas taxinomicamente, seguindo os critérios de Guimarães ${ }^{7}$, Lima ${ }^{10}$ e Johnson ${ }^{8}$.

A Tabela 1 exibe o número total de pulgas capturadas de 18 exemplares de raposas, e identificadas como: Rhopalopsyllus lutzi lutzi (Baker, 1904), Pulex irritans Linnaeus, 1758, Ctenocephalides canis (Curtis, 1826), Ctenocephalides felis felis (Bouché, 1835) e Xenopsylla cheopis (Rothschild, 1903).

Tabela 1 - Espécies e número de pulgas capturadas em Cerdocyon thous, de Jacobina, Bahia, no período de julho/ setembro 1998.

\begin{tabular}{lrrr} 
& \multicolumn{2}{c}{ Sexo } & \multicolumn{1}{c}{} \\
\cline { 2 - 3 } Espécies & $\mathrm{M}$ & 103 & Total \\
\hline Rhopalopsyllus lutzi lutzi & 33 & 4 & 136 \\
Pulex irritans & 7 & 1 & 1 \\
Ctenocephalides canis & 1 & 0 & 2 \\
Ctenocephalides felis felis & 1 & 1 & 1 \\
Xenopsylla cheopis & 1 & 109 & 1 \\
\hline Total & 43 & & 152 \\
\hline
\end{tabular}

A espécie mais freqüente foi Rhopalopsyllus lutzi lutzi, e embora já assinalada em outras áreas infestando diversas espécies de carnívoros, tratase do primeiro registro para o estado da Bahia (PM Linardi, LR Guimarães: dados não publicados).

É interessante enfatizar a presença das espécies de pulgas do homem, e de mamíferos domésticos, respetivamente, Pulex irritans, Cetenocephalides canis e Ctenocephalides felis felis, parasitando a raposa, o que poderia sugerir a existência de uma possível conexão ecoepidemiológica do referido hospedeiro silvestre de Leishmania chagasicom o ambiente doméstico, oportunidade em que poderia infestar-se pelas pulgas próprias do homem ou e animais domésticos, quando da incursão dos cães nos ecotópos naturais silvestres próprios da raposa.

A presença de Xenopsylla cheopis, uma pulga habitual de ratos, atesta a favor do intercâmbio e de uma provável comensalidade ecológica do roedor com a raposa, ambos suspeitos como possíveis reservatórios primários de Leishmânia chagasi.

Uma vez que as pulgas têm sido pouco investigadas como possíveis transmissores de leishmanioses, principalmente a visceral, tornase interessante uma pesquisa sobre o real papel dos sifonápteros como vetores de leishmânias, inclusive através de técnicas de PCR, como aliás já delineada em nosso laboratório. 


\section{AGRADECIMENTOS}

Os autores agradecem ao Prof. Pedro Marcos Linardi, do Departamento de Parasitologia do Instituto de Ciências Biológicas da Universidade Federal de Minas Gerais, pela revisão da identificação das pulgas. Ao técnico Antônio Carlos dos Santos, do Laboratório de Parasitologia e Entomologia do Centro de Pesquisas Gonçalo Moniz/FIOCRUZ, pela ajuda na captura e exame das raposas.

\section{REFERÊNCIAS BIBLIOGRÁFICAS}

1. Alencar JE. Leishmaniose canina. Contribuição para o estudo da epidemiologia do calazar no Brasil. Tese de Livre Docência, Universidade Federal do Ceará, Fortaleza, CE, 1959.

2. Beard CB, Butler JF, Orshar EC. Growth characterization and host-parasite relationship of Leptomonas pulexsimulantis $n s p$, a trypanosomatid flagellate of the flea Pulex simulans. Journal of Parasitology 75: 658-668, 1989.

3. Bibikova VA. Conteporany views on the interalationships between fleas and the pathogens of human and animal diseases. Annual Review of Entomology 22: 23-32, 1977.

4. Courtenay O, Santana EW, Johnson PJ, Vasconcelos IAB, Vasconcelos AW. Visceral leishmaniasis in the hoary zorro Dusicyon vetulus: a case of mistaken identity. Transactions of the Royal Society of Tropical Medicine and Hygiene 90:498-502, 1996.

5. Daoust RA. XV. Pathogens of Siphonaptera (Fleas). In: Roberts DW, Daoust RA, Wraight SP (eds) Bibliograph on Pathogens of Medically Important Arthropods, World Helath Organization, Genéve, p. 249-253, 1983.

6. Deane LM. Leishmaniose visceral no Brasil. Estudos sobre reservatórios e transmissores realizados no Estado do Ceará. Serviço Nacional de Educação Sanitária, Rio de Janeiro, RJ, 1956.

7. Guimarães LR. Contribuição à epidemiologia da peste endêmica no nordeste do Brasil e Estado da Bahia.
Estudo das pulgas encontradas nessa região. Revista Brasileira de Malariologia e Doenças Tropicais 24: 95164, 1972.

8. Jonhson PT. A classification of the Siphonaptera of South America. Memoirs of the Entomological Society of Washington n. 5, Washington, DC, USA, 1957.

9. Lainson R, Dye C, Shaw JJ, Macdonald D, Courtnenay O, Souza AA, Silveira FT. Amazonian visceral leishmaniasis: distribuition of the vector Lutzomyia longipalpis (Lutz e Neiva) in relation to the fox Cerdocyon thous (L.) and the efficiency of this reservoir host as a source of infection. Memorias do Instituto Oswaldo Cruz 85:135-137, 1957

10. Lima AC. Suctoria. In: Insetos do Brasil. Escola Nacional de Agronomia. Série Didática no.6, Rio de Janeiro, RJ, vol. IV, p.17-71, 1943.

11. Pacheco RS, Marzochi MCA, Pires MQ, Brito CMM, Madeira MF, Barbosa-Santos GO. Parasite genotypically related to a monoxenous trypanosomatid of dog's flea causing opportunistic infection in an HIV positive patient. Memorias do Instituto Oswaldo Cruz 93:531-537,1998.

12. Sherlock IA. Nota sobre a transmissão da leishmaniose visceral no Brasil. Revista Brasileira de Malariologia e Doenças Tropicais 16: 19-26, 1964. 